OPEN ACCESS

Edited by:

Jens Staal,

Ghent University, Belgium

Reviewed by:

Mehdi Kabbage,

University of Wisconsin-Madison

United States

Shaojun Dai,

Northeast Forestry University, China

${ }^{*}$ Correspondence:

Justice Norvienyeku

jk_norvienyeku@fafu.edu.cn

Zonghua Wang

wangzh@fafu.edu.cn

${ }^{\dagger}$ These authors have contributed equally to this work

Specialty section:

This article was submitted to Plant Microbe Interactions,

a section of the journal

Frontiers in Plant Science

Received: 04 March 2018

Accepted: 15 June 2018

Published: 08 August 2018

Citation:

Abdul W, Aliyu SR, Lin L, Sekete M,

Chen X, Otieno FJ, Yang T, Lin Y,

Norvienyeku J and Wang Z (2018)

Family-Four Aldehyde

Dehydrogenases Play an Indispensable Role

in the Pathogenesis of Magnaporthe

oryzae. Front. Plant Sci. 9:980.

doi: 10.3389/fp/s.2018.00980

\title{
Family-Four Aldehyde Dehydrogenases Play an Indispensable Role in the Pathogenesis of Magnaporthe oryzae
}

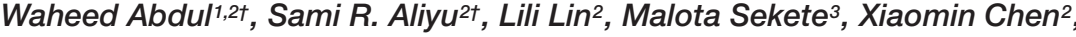 \\ Frankline J. Otieno', Tao Yang ${ }^{2}$, Yahong Lin ${ }^{1}$, Justice Norvienyeku ${ }^{1,2 *}$ and \\ Zonghua Wang ${ }^{1,2,4 *}$
}

${ }^{1}$ Fujian University Key Laboratory for Functional Genomics of Plant Fungal Pathogens, College of Life Sciences, Fujian Agriculture and Forestry University, Fuzhou, China, ${ }^{2}$ Fujian and Taiwan Joint Center for Ecological Control of Crop Pests, College of Plant Protection, Fujian Agriculture and Forestry University, Fuzhou, China, ${ }^{3}$ Department of Crop Science, Faculty of Agriculture, National University of Lesotho, Lesotho, Southern Africa, ${ }^{4}$ Institute of Oceanography, Minjiang University, Fuzhou, China

The oxidative degradation of lipids through lipid peroxidation processes results in the generation of free fatty acid radicals. These free radicals including reactive oxygen species (ROS) serve as a substrate for generating reactive aldehydes. The accumulation of free fatty acid radicals, ROS, and reactive aldehydes in cell compartments beyond physiological threshold levels tends to exert a damaging effect on proximal membranes and distal tissues. Living organisms deploy a wide array of efficient enzymes including superoxide dismutase (SOD), catalase (CAT), peroxidase (POD), and aldehyde dehydrogenases $(A L D H S)$ for scavenging reactive molecules and intermediates produced from membrane lipid peroxidation events. Although the contributions of $S O D, C A T$, and $P O D$ to the pathogenesis of microbial plant pathogens are well known, the influence of $A L D H$ genes on the morphological and infectious development of plant pathogenic microbes is not well understood. In this study, we deployed RNA interference (RNAi) techniques and successfully silenced two putative familyfour aldehyde dehydrogenase genes potassium-activated aldehyde dehydrogenase $(\mathrm{MoKDCDH})$ and delta-1-pyrrorine-5-carboxylate dehydrogenase $(\mathrm{MoP} 5 \mathrm{CDH})$ in the rice blast pathogen Magnaporthe oryzae. The results obtained from the phenotypic analysis of individual knock-down strains showed that the RNAi-mediated inactivation of $M O K D C D H$ and MoP5CDH triggered a significant reduction in conidiogenesis and vegetative growth of $\Delta M o k d c d h$ and $\Delta M o p 5 c d h$ strains. We further observed that downregulating the expression of $M O K D C D H$ and $M O P 5 C D H$ severely compromised the pathogenesis of the rice blast fungus. Also, the disruption of $M O K D C D H$ and $\mathrm{MoP} 5 \mathrm{CDH}$ M. oryzae undermined membrane integrity and rendered the mutant strains highly sensitive to membrane stress inducing osmolytes. However, the MoKDCDH and $\mathrm{MoP} 5 \mathrm{CDH}$ knock-down strains generated in this study displayed unaltered cell wall integrity and thus suggested that family-four ALDHs play a dispensable role in 
enforcing cell wall-directed stress tolerance in $M$. oryzae. From these results, we deduced that family-four ALDHs play a conserved role in fostering membrane integrity in $M$. oryzae possibly by scavenging reactive aldehydes, fatty acid radicals, and other alcohol derivatives. The observation that downregulating the expression activities of MoKDCDH had a lethal effect on potential mutants further emphasized the need for comprehensive and holistic evaluation of the numerous $A L D H s$ amassed by the rice blast fungus for their possible engagement as suitable targets as antiblast agents.

Keywords: aldehyde dehydrogenase, free fatty acid radicals, lipid peroxidation, aldehydes, Magnaporthe oryzae

\section{INTRODUCTION}

Aldehydes are intermediates in several fundamental metabolic pathways, including the synthesis of carbohydrates, vitamins, steroids, amino acids, and lipids (Kirch et al., 2004). Aldehydes also accumulate in tissues in response to environmental stresses, including salinity, dehydration, desiccation, cold, and heat shock (Feder and Hofmann, 1999). Aldehyde molecules are reactive at excessive physiological concentrations and hence tend to negatively impact on cell growth, yield, seed survival, and membrane integrity (Kotchoni et al., 2010). Living organisms tightly regulate the cellular aldehyde level by limiting membrane peroxidation events by exploring antioxidant properties of either superoxide dismutase $(S O D)$, catalase $(C A T)$, or peroxidase (POD) (Schnitzer et al., 2007; Hossain et al., 2015). Furthermore, the direct regulation of cellular aldehyde levels involves enzymatic activities of aldehyde dehydrogenases (Singh et al., 2013).

Aldehyde dehydrogenases (ALDHs) are a group of evolutionarily conserved polymorphic enzymes (Zhu et al., 2014). ALDHs belong to a superfamily of $\mathrm{NADP}^{+}$-dependent enzymes that are involved in the irreversible oxidation of endogenous and exogenous aldehydes to their corresponding carboxylic acids (Zhang et al., 2012; Luo et al., 2015; Buchman and Hurley, 2017). A total of 19 dehydrogenase-encoding genes have been recorded in the human genome. Insights gained from these previous studies showed that human $A L D H$ s play critical cellular and biological roles including detoxification of aldehydes, cell proliferation, peroxidation of membrane lipid, protection of tissues against hyperosmotic pressure, and inhibition of tumors and cancers (Januchowski et al., 2013; Morgan and Hurley, 2015).

The research evidence currently available also showed that $A L D H$ s promote abiotic stress tolerance, facilitate the restoration of male sterility, and regulate embryo development and seed viability as well as maturation in different plant species including rice (Kotchoni et al., 2010; Gill, 2014). Although plants have several distinct types of $A L D H$ s, information regarding their individual and collective biological role is limited and requires further investigations (Kotchoni et al., 2006; Stiti et al., 2011).

Previous studies have also shown that reactive oxygen species (ROS) and other reactive molecules including aldehydes play dual functions during host-pathogen interaction. Plants accumulate free fatty acid radicals and other derivatives of membrane lipid peroxidation processes as a significant and earliest pathogen-triggered immune (PTI) response (Palukaitis and Carr, 2008; Heller and Tudzynski, 2011; Ranf et al., 2011; Smith and Heese, 2014; Lehmann et al., 2015). Under this situation, plants generate free radicals and reactive aldehydes as direct reactive substrates to kill pathogens or as secondary defence signaling molecules for activating durable host resistance against invading pathogens (Norvienyeku et al., 2017). Pathogens, in contrast, generate and secrete a wide range of free fatty acid radicals, ROS, reactive aldehydes, and alcohol derivatives that are functionally and structurally similar to reactive molecules produced by plants as virulence factors during host-pathogen interactions (Heller and Tudzynski, 2011; Huang et al., 2011). The majority of these reactive molecules have a damaging effect on cell wall and cell membranes (Thannickal and Fanburg, 2000). During host-pathogen interaction, microbial pathogens secretes reactive molecules into host cells to successfully suppress host immunity (Koop, 2006; Bryan et al., 2012).

Many research findings have shown that plants, as well as microbial pathogens, deploy $S O D, C A T$, and $P O D$ for ROS scavenging. Although aldehydes are known as one of the most diverse lipid oxidation products (LOPs), the knowledge on aldehyde scavenging activities of aldehyde dehydrogenases during growth, reproduction, and infectious development is not extensively studied in filamentous phyto-pathogenic fungi.

In our previous study, we showed that the filamentous ascomycete fungus Magnaporthe oryzae possesses a total of 16 $A L D H$ s in its genome (Norvienyeku et al., 2017). ALDH genes are prone to gene duplication events (Zhang et al., 2012). Moreover, records from our previous studies showed that the 12 of the 16 $A L D H$ genes identified in $M$. oryzae experienced gene duplication while the remaining 4 genes, including methylmalonatesemialdehyde dehydrogenase (MoMSDH/MoMMSDH), betaine aldehyde dehydrogenase1 (MoBADH1), potassium-activated aldehyde dehydrogenase $(M o K D C D H)$, and delta-1-pyrrorine5 -carboxylate dehydrogenase $(\mathrm{MoP} 5 \mathrm{CDH})$, exist as single copies. We also showed that the targeted deletion of $M o M S D H$ severely compromised sporulation, germination, appressorium morphogenesis, redox homeostasis, and pathogenesis of $\Delta$ Momsdh strain (Norvienyeku et al., 2017).

The additional research also showed that the disruption of succinate semialdehyde dehydrogenase 2 (MoSSADH2) in the rice blast fungus equally triggered a significant reduction in growth and completely abolished sporulation in defective strains; 
all these studies showed that $A L D H$ s might assume roles beyond detoxification of endogenously generated and exogenously translocated reactive aldehydes.

In this study, we silenced the remaining single copy of $A L D H$ genes ( $M o K D C D H, M o P 5 C D H$, and $M o B A D H 1)$ using RNA interference (RNAi) strategy. We proceeded further to evaluate growth, sporulation, and infection characteristics of the $\Delta M o k d c d h$ and $\Delta M o p 5 c d h$ strains. The corresponding results obtained from this investigation showed that the silencing of $M o K D C D H$ and $M o P 5 C D H$ exerts adverse effects on growth, sporulation, and pathogenesis of $M o K D C D H$ and $M o P 5 C D H$ knock-down strains. However, for some unknown reason, attempts aimed at silencing $M o B A D H 1$ were unsuccessful.

\section{RESULTS AND DISCUSSION}

\section{Domain Homology and Architecture of M. oryzae Aldehyde Dehydrogenases}

Aldehyde dehydrogenases are evolutionarily conserved and ubiquitously present in all living organisms across kingdoms. Most members of the $A L D H$ superfamily are associated with high incidences of gene duplication (Brocker et al., 2013). In our previous investigation, we identified a total of $16 A L D H$ (MoALDH)-encoding genes in the rice blast fungus genome and further demonstrated that 12 of the 16 ALDHs identified in M. oryzae have multiple copies (Norvienyeku et al., 2017).

To provide insights into inherent cladistic relationship prevailing between the respective MoALDHs, we performed Pfam $^{1}$ and SMART $^{2}$-based assisted domain prediction analysis for all the $16 \mathrm{M}$. oryzae ALDHs. The results obtained from domain prediction analysis showed that all the $16 \mathrm{MoALDHs}$ possessed the conserved Aldedh domain (Figure 1A), and given this observation, we accordingly inferred that the Aldedh domain likely represents a basic but indispensable genetic parameter that defines aldehyde-catalyzing capabilities of the respective Aldedhs. We additionally performed phylogenetic and domain homology analyses using amino acid (aa) sequences of the Aldedh-domain region of all the $16 \mathrm{M}$. oryzae ALDHs. The phylogenetic cladogram obtained from this examination revealed that based on the domain sequence homology, MoALDHs could be grouped into nine distinct and diverse subfamilies (Figure 1B). The high and diverse number of clusters recorded from the bootstrap maximum-likelihood analysis conducted in this study could be attributed to the fact that the traditional classification of $A L D H s$ was based on their requirement for $\mathrm{NAD} / \mathrm{NADP}^{+}$as a reaction co-factor rather than conserved Aldedh domain (Perozich et al., 1999). Further, the subfamilylevel mapping was carried out by conducting homologous and orthologous BLASTP search with domain sequences of respective $M o A L D H s$ in the human genome database $e^{3}$ and $A L D H^{4}$ website,

\footnotetext{
${ }^{1}$ http://pfam.xfam.org/search/sequence

${ }^{2}$ http://smart.embl-heidelberg.de

${ }^{3}$ https://www.genenames.org/cgi-bin/search

${ }^{4}$ http://www.aldh.org/website/aldh/superfamily.php
}

Arabidopsis genome database ${ }^{5}$, and rice genome database ${ }^{6}$. The subfamily level classification results showed that the 16 $M o A L D H$ genes belong to eight different $A L D H$ subfamilies (Table 1). Since different members of the $A L D H$ family acts on different types of aldehydes as substrates (Jimenez-Lopez et al., 2016), we subsequently posited that phylogeny, domain structure, and wide subfamily-level divergence exhibited by the various $M o A L D H$ genes are likely a reflection of their functional divergence.

\section{Generation of MoKDCDH and MoP5CDH Knock-Down Mutants}

In our previous studies, we showed that the expression activities of $M o K D C D H$ and $M o P 5 C D H$ fluctuated significantly during tissue invasion and colonization stages of the rice blast fungus. To assess the influence of these substrate-specific $A L D H$ genes on the physiological and infectious development of the rice blast fungus, we initially deployed homologous recombination strategy in an attempt to generate $A L D H$-targeted gene deletion strains for $M o K D C D H$ and $M o P 5 C D H$. However, we could not obtain positive gene replacement mutants for the two $A L D H$ genes even after a series of repeated protoplast transformations followed by the screening of more than 2000 potential candidates for each gene. We subsequently adopted RNAi approach to generate knock-down mutants by transforming $M o K D C D H$ and $M o P 5 C D H$ knock-down plasmid vector construct into $M$. oryzae Guy11 strain. The corresponding results obtained from a qPCR-mediated screening of prospective $M o K D C D H$ and $M o P 5 C D H$ gene knock-down transformants revealed six (6) and three (3) independent knock-down mutants for $M o K D C D H$ and $M o P 5 C D H$, respectively (Figures 2A,B). Also to assess the influence (off-target effect) of RNAi-mediated disruption of $M o K D C D H$ and $M o P 5 C D H$ on other members of the ALDH family, we monitored the transcriptional activities of individual members of $M$. oryzae ALDH superfamily in each independent knock-down strain relative to the wild-type strain using qPCR. Results obtained from these analyses revealed that the disruption of $M o K D C D H$ and $M o P 5 C D H$ did not significantly alter the expression pattern of other members of the $A L D H$ superfamily (Supplementary Figure S1) indicating that the silencing of $M o K D C D H$ and $M o P 5 C D H$ had no off-target effect on other ALDHs in M. oryzae.

Our studies revealed that $M o K D C D H$ shared the same subfamily (family-four) with $M o P 5 C D H$, and records have shown that the deletion of MoP5CDH ortholog DmP5CDh1 in Drosophila melanogaster triggered proline accumulation and swollen mitochondria and subsequently resulted in larval and pupal lethality (Koštál et al., 2012). This knowledge coupled with our inability to generate $\mathrm{MoKDCDH}$ and $\mathrm{MoP} 5 \mathrm{CDH}$ targeted gene replacement mutants subsequently informed our speculative conclusion that $M o K D C D H$ and $M o P 5 C D H$ likely play indispensable roles in the survival of the rice blast fungus.

\footnotetext{
${ }^{5}$ https://www.arabidopsis.org/

${ }^{6}$ https://shigen.nig.ac.jp/rice/oryzabase/
} 


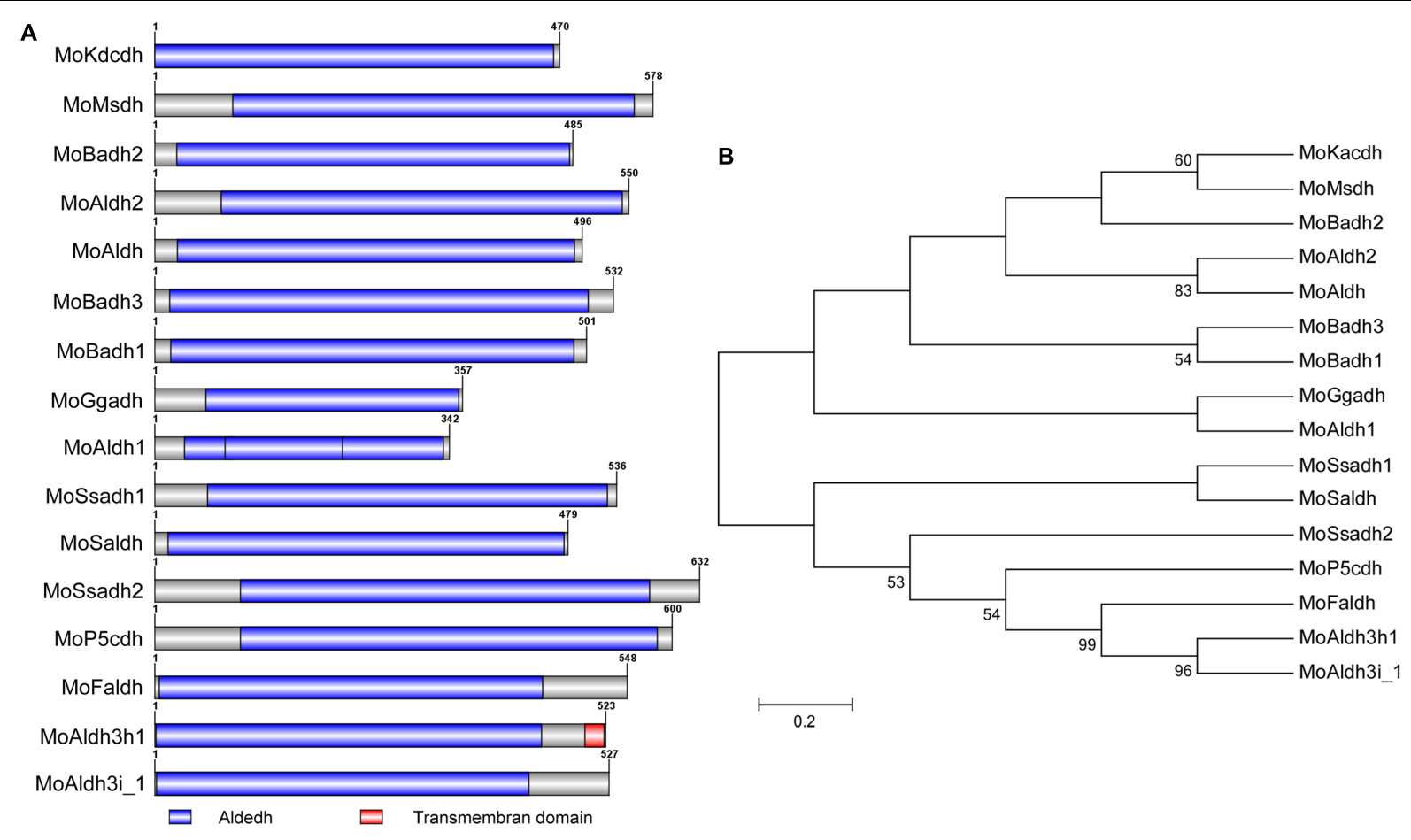

FIGURE 1 | Domain architecture and domain base phylogeny of MOALDHs. (A) Structure dynamics of the conserved Aldedh-domain predicted for the 16 ALDHs identified in M. oryzae genome. (B). Domain sequence-mediated clustering and maximum-likelihood phylogeny of all the $16 \mathrm{M}$. oryzae aldehyde dehydrogenases tested with 1,000 bootstrap replicates.

\section{Family-Four ALDHs Are Required for the Survival, Growth, Sporulation, and Pathogenesis of M. oryzae}

Upon successful silencing of $M o K D C D H$ and $M o P 5 C D H$, we assayed the contribution of $M o K D C D H$ and $M o P 5 C D H$ to the vegetative growth and morphological development of $M$. oryzae by culturing $M o K D C D H$ and $M o P 5 C D H$ knock-down mutants and the wild-type strain on complete media (CM) for 10 days under $28^{\circ} \mathrm{C}$ before proceeding to measure the colony diameter of respective mutants. The results obtained from these examinations showed that downregulating the activities of $M o K D C D H$ and $M o P 5 C D H$ resulted in a corresponding reduction in the vegetative growth of the individual gene knock-down mutants (Figures 3A-D). The seeming correlation observed between the expression levels of $M o K D C D H$ and $M o P 5 C D H$, their respective knock-down lines, and the magnitude of growth reduction informed our conclusion that the silencing of $M o K D C D H$ and $M o P 5 C D H$ triggered the growth defects exhibited by the $\Delta M o k d c d h$ and $\Delta M o p 5 c d h$ strains.

To further unravel the influence of $M o K D C D H$ and $M o P 5 C D H$ on the generation of asexual spores in the rice blast fungi, we instituted relevant sporulation assays to examine the quantum and morphology of asexual spores generated by $M o K D C D H$ and $M o P 5 C D H$ knock-down mutants relative to the wild type. The results obtained from these investigations showed that the RNAi-mediated silencing of $M o K D C D H$ and $\mathrm{MoP} 5 \mathrm{CDH}$ drastically compromised sporulation characteristics of both $M o K D C D H$ and $M o P 5 C D H$ knock-down mutants (Figures 3E,F). We also showed that $M o K D C D H$ exclusively played a role in modulating conidia morphogenesis; hence, $M o K D C D H$ knock-down mutants displayed abnormal conidium morphogenesis, conidium septation, germination, and appressorium morphogenesis (Figure 3G). Conversely, $M o P 5 C D H$ knock-down strains displayed intact conidia and appressorium morphology with no visible septation and germination defects (Figure $\mathbf{3 H}$ ), and we further observed that downregulating the expression activities of $M o K D C D H$ to levels below 50\% had lethal effect. Hence, the resultant mutants displayed short lifespan (could not be stored beyond 3 months). From these results, we inferred that $M o K D C D H$ likely mediates conidia and appressoria morphogenesis in the rice blast fungus through direct or indirect regulation independent pathways that are not under the collective influence of the family-four $A L D H$ s. The additional results derived conidia-mediated infection assays showed that both $M o K D C D H$ and $M o P 5 C D H$ knock-down strains with substantially reduced expression activities failed to induce blast infection, and as a result, susceptible rice seedlings sprayed inoculated independently with conidia from $M o K D C D H$ and $M o P 5 C D H$ knock-down strains in suspension displayed reduced lesions (Figures 3I,J).

$M o K D C D H$ and $M o P 5 C D H$ are family-four aldehyde dehydrogenases, and we showed that $M o K D C D H$ and MoP5CDH knock-down mutants with $<50 \%$ expression activity were nonpathogenic. Growth records obtained from this 
TABLE 1 | Family level classification of $M$. oryzae aldehyde dehydrogenase (MOALDHS).

\begin{tabular}{|c|c|c|c|}
\hline Family & Accession No. & Actual Name & Given Name \\
\hline Family 1 & Mgg_03900 & Aldehyde dehydrogenase & $\mathrm{ALDH}$ \\
\hline \multirow[t]{2}{*}{ Family 2} & Mgg_13331 & $\begin{array}{l}\text { Gamma-glutamyl-gamma- } \\
\text { aminobutyraldehyde } \\
\text { dehydrogenase }\end{array}$ & GGADH \\
\hline & Mgg_06551 & Aldehyde dehydrogenase & ALDH1 \\
\hline \multirow[t]{3}{*}{ Family 3} & Mgg_07270 & Fatty aldehyde dehydrogenase & FALDH \\
\hline & Mgg_00719 & Aldehyde dehydrogenase $3 \mathrm{H} 1$ & ALDH3H1 \\
\hline & Mgg_07890 & Aldehyde dehydrogenase & ALDH3I_1 \\
\hline \multirow[t]{2}{*}{ Family 4} & Mgg_05814 & $\begin{array}{l}\text { Potassium-activated aldehyde } \\
\text { dehydrogenase }\end{array}$ & $\mathrm{KACDH}$ \\
\hline & Mgg_17513 & $\begin{array}{l}\text { Delta-1-pyrrorine-5-carboxylate } \\
\text { dehydrogenase }\end{array}$ & $\mathrm{P} 5 \mathrm{CDH}$ \\
\hline \multirow[t]{4}{*}{ Family 5} & Mgg_00652 & Salicylaldehyde dehydrogenase & SALDH \\
\hline & Mgg_01230 & $\begin{array}{l}\text { Succinate-semi aldehyde } \\
\text { dehydrogenase }\end{array}$ & SSADH1 \\
\hline & Mgg_02766 & $\begin{array}{l}\text { Succinate-semi aldehyde } \\
\text { dehydrogenase }\end{array}$ & SSADH2 \\
\hline & Mgg_05008 & Aldehyde dehydrogenase & ALDH2 \\
\hline Family 6 & Mgg_01606 & $\begin{array}{l}\text { Methylmalonate-semialdehyde } \\
\text { dehydrogenase }\end{array}$ & $\mathrm{MSDH}$ \\
\hline Family 9 & Mgg_09456 & $\begin{array}{l}\text { Betaine aldehyde } \\
\text { dehydrogenase }\end{array}$ & $\mathrm{BADH} 1$ \\
\hline \multirow[t]{2}{*}{ Family 10} & Mgg_03263 & $\begin{array}{l}\text { Betaine aldehyde } \\
\text { dehydrogenase }\end{array}$ & $\mathrm{BADH} 2$ \\
\hline & Mgg_01991 & $\begin{array}{l}\text { Betaine aldehyde } \\
\text { dehydrogenase }\end{array}$ & $\mathrm{BADH} 3$ \\
\hline
\end{tabular}

study showed that the silencing of $M o K D C D H$ and $M o P 5 C D H$ also caused a significant reduction in vegetative growth and adequately support our conclusion that the full participation of family-four aldehyde dehydrogenases (potassium-activated aldehyde dehydrogenase and delta-1-pyrrorine-5-carboxylate dehydrogenase) is likely necessary for growth, virulence, and pathogenicity of the rice blast fungus during host-pathogen interaction. This position is firmly supported by previous research findings, which equally showed that potassiumactivated aldehyde dehydrogenase in Colletotrichum acutatum functions as a pathogenicity factor that promoted disease development (Guidarelli et al., 2011).

\section{Response of MoKdcdh and MoP5Cdh Knock-Down Mutants to Oxidative Stress}

Since aldehyde dehydrogenases are inherently involved in the peroxidation of membrane lipids coupled with the fact that the peroxidation of membrane lipid enhances membrane integrity and bolsters cell membrane against oxidative stress.

We subsequently decided to assess the response of $M o K D C D H$ and $M o P 5 C D H$ knock-down mutants by culturing respective knock-down lines along with the wild-type strain on CM supplemented with oxidative and reductive stress-inducing agents including acetaldehydes, alcohol, SDS, ROS, and DTT. The individual knock-down mutants were highly sensitive to
SDS, ROS, and $\mathrm{NaCl}$ (oxidative stress) (Trotter and Grant, 2002; Vaidyanathan et al., 2003; Furukawa et al., 2017; Conrad et al., 2018) and MoP5CDH and DTT (reductive stress) (Trotter and Grant, 2002; Valadi et al., 2004). From these results, we concluded that $M o K D C D H$ and $M o P 5 C D H$ promote oxidative and reductive stress tolerance of $M$. oryzae.

Furthermore, $M o K D C D H$ and $M o P 5 C D H$ knock-down strains displayed higher sensitivity toward alcohol and ROS (Figures 4A-D). Records currently available showed that $\mathrm{P} 5 \mathrm{CDH}$ enforces membrane integrity and oxidative stress tolerance by catalyzing the transformation of delta-1-pyrroline-5-carboxylate derived from proline or ornithine into glutamate (HirayamaKurogi et al., 2017). Therefore, we subsequently posited that the two family-four aldehyde dehydrogenases retain and play their conserved role in the detoxification of ROS, reactive aldehydes, and other alcohol derivatives generated during the morphological, physiological, and infectious development of M. oryzae.

\section{CONCLUSION}

Aldehyde dehydrogenases $(A L D H s)$ are an evolutionarily conserved group of NAD/NADP-dependent multigenic enzymes that are involved in the irreversible oxidation of endogenous and exogenous reactive aldehydes to their nontoxic corresponding carboxylic acids (Dong et al., 2017; Li et al., 2017; Norvienyeku et al., 2017). Members of the $A L D H$ superfamily are grouped into subfamilies based on the type of conserved amino acid residue clusters found around the enzyme-binding sites (Guo et al., 2017; Jia et al., 2017; Muñoz-Clares et al., 2017; Missihoun et al., 2018). Currently, more than 25 ALDH subfamilies have been identified in different organisms, plant $A L D H$ s have been characterized into the subfamily level in different plant species including Solanum lycopersicum, Arabidopsis thaliana, C. reinhardtii, O. tauri, the moss P. patens, and Zea mays (Kirch et al., 2004; Wood and Duff, 2009; Kotchoni et al., 2010). In humans, a total of $19 A L D H s$ have been identified and classified into 11 families and well characterized (Vasiliou et al., 2004; Zhang et al., 2012). However, fungi $A L D H$ s have not received the needed attention with regard to classification and characterization. In this study, the $16 \mathrm{MoALDH}$ s previously identified in M. oryzae were successfully classified into seven families. In contrast to humans, we showed that $M$. oryzae family-four $A L D H s$ consists of two members, $M o P 5 C D H$, an ortholog of human delta-1-pyrroline-5-carboxylate dehydrogenase, and ALDH4A1 in addition to potassium-activated aldehyde dehydrogenase ( $\mathrm{He}$ and DiMario, 2011; Yao et al., 2013). In humans, family-four $A L D H$ inactivity has been associated with seizures, mental retardation, and cellular stress tolerance (Pérez-Arellano et al., 2010). It has also been shown that the deletion of family $A L D H$ in Drosophila melanogaster triggered larval and pupal lethality (Wan et al., 2015).

Our studies showed that the successful RNAi-mediated silencing of family-four MoALDHs severely compromised growth, sporulation, stress tolerance, and pathogenicity of $M o K D C D H$ and $M o P 5 C D H$ knock-down mutants and 

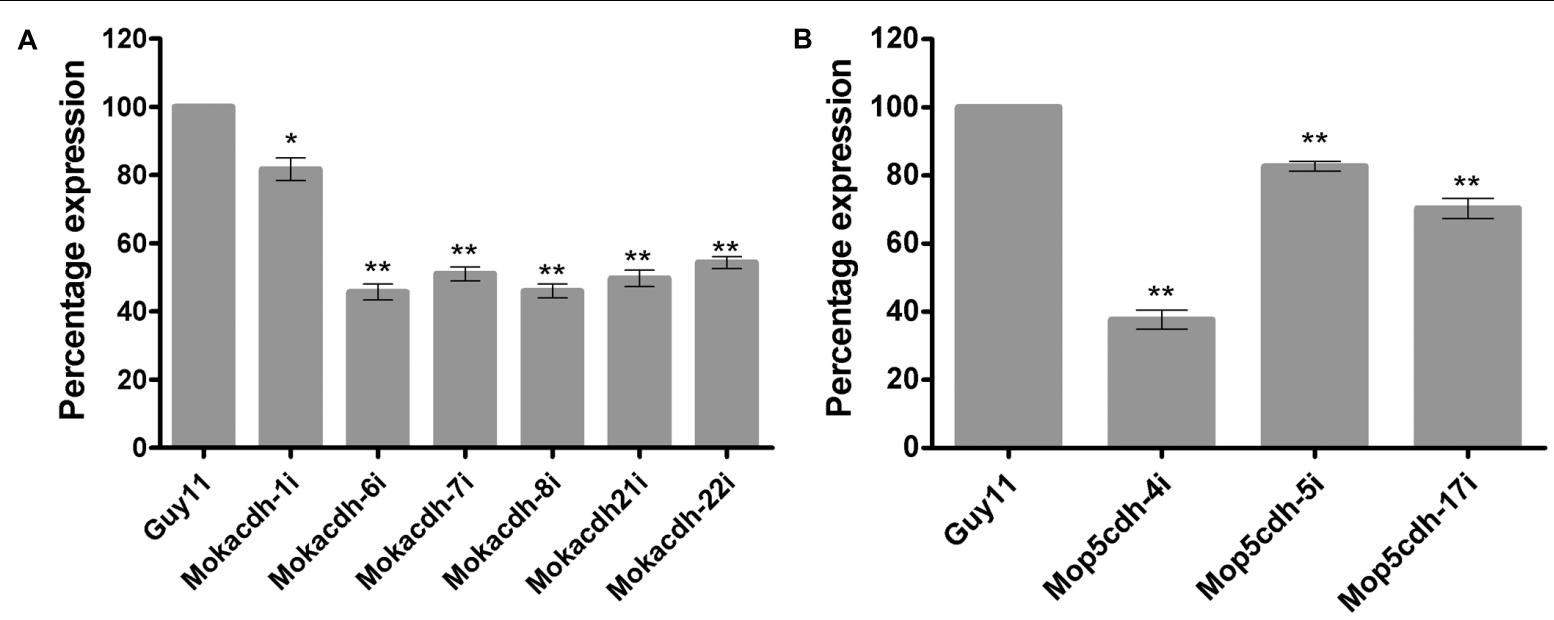

FIGURE 2 | Percentage (\%) fold expression of $M O K D C D H$ and $M O P 5 C D H$ in respective knock-down mutants. (A) The expression level of $M o K D C D H$ in six different independent knock-down mutants of $M O K D C D H$. (B) The fold expression of MoP5CDH in the three independent MoP5CDH knock-down mutants. The quantitative real-time PCR (qRT-PCR) data were computed with Microsoft Excel spread sheet in conjuction with graphpad prism6. The error bars represent mean \pm SD, whereas single and double asterisks "*" represent a significant reduction in the fold expression of MoKDCDH and MoP5CDH in their respective knock-down strains.

Consistent values were obtained with five independent biological replications and three technical replicates for each independent qRT-PCR experiment.

subsequently confirmed previous research submissions that $P 5 C D H$ promoted sporulation and virulence of fungal pathogens (Yao et al., 2013). These findings further showed that a complete evaluation of whole-genome aldehyde dehydrogenases in the model plant pathogenic fungi would tremendously contribute to enhance our knowledge with regard to the fundamental role of $A L D H$ s during the host-pathogen interaction and their deployment as potential targets for fungicide development.

\section{MATERIALS AND METHODS}

\section{Strains}

Magnaporthe oryzae wild-type strain Guy11 used in this study to generate mutant $A L D H$ deletion mutants was kindly provided by Dr. Didier Tharreau (CIRAD, Montpellier, France). Competent cells used in this research for the propagation of the constructed plasmids were prepared from Escherichia coli (E. coli) strain DH5 $\alpha$.

\section{Growth Media}

The Guyl1 strain used in this study to generate $A L D H$ mutants were cultured under an optimum temperature of $28^{\circ} \mathrm{C}$ on complete medium (CM: $0.6 \%$ yeast extract, $0.6 \%$ casein hydrolysate, $1 \%$ sucrose, and $1.5 \%$ agar). The E. coli strain $\mathrm{DH} 5 \alpha$ used in this study to generate competent cells was cultured on lysogeny broth (LB) medium prepared by dissolving $10 \mathrm{~g}$

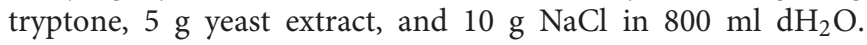
The $\mathrm{pH}$ adjusted to 7 by adding $1 \mathrm{M} \mathrm{NaOH}$ in a dropwise manner before adding up more deionized water to make up to the desired volume of $1 \mathrm{~L}$ with $\mathrm{dH}_{2} \mathrm{O}$. Both $\mathrm{CM}$ and $\mathrm{LB}$ growth media were sterilized by autoclaving at $121^{\circ} \mathrm{C}$ for $20 \mathrm{~min}$.

\section{The Chemicals}

All reagents and chemicals, as well as kits used in this study, are certified analytical grade, which are purchased from certified suppliers including Sigma-Aldrich, Amresco, Chromotek, and Roche.

\section{Primers and Plasmids}

Nucleotide sequences for the individual $A L D H$ s were acquired from comparative $M$. oryzae genome database established by Broad Institute ${ }^{7}$. The primers used for amplifying respective sequences to generate RNAi knock-down mutant strains were designed with the BACON primer designer. BGI, Shenzhen, sequenced all designed primers; a pTE11 plasmid containing neuromycine as the selective marker was used as a fusion vector for generating RNAi silence mutant strains.

\section{Protoplast Preparation}

The Guy11 isolates were cultured or grown in liquid for 4 days in a shaking incubator at a temperature of $27.5^{\circ} \mathrm{C}$ at $120 \mathrm{rpm}$. Media were carefully drained off leaving the colonies behind, and the cultured colonies were then macerated by grinding. The grounded tissues were re-suspended in a fresh liquid CM and incubated in a shaking incubator operating at a speed of $120 \mathrm{rpm}$ overnight at a temperature of $27.5^{\circ} \mathrm{C}$. Mycelium were harvested from the liquid CM by filtering and washed with sterilized double deionized water followed by $1 \mathrm{M}$ sorbitol. The tissues were then dried with the filter paper and re-suspended in $40 \mathrm{ml}$ of $1 \mathrm{M}$ sorbitol containing $100 \mathrm{mg}$ lysing enzyme from Trichoderma harzianum and incubated for $3 \mathrm{~h}$ under $30^{\circ} \mathrm{C}$ at $60 \mathrm{rpm}$; protoplasting was observed on an hourly basis. Generated

\footnotetext{
${ }^{7}$ http://www.broadinstitute.org/
} 
A

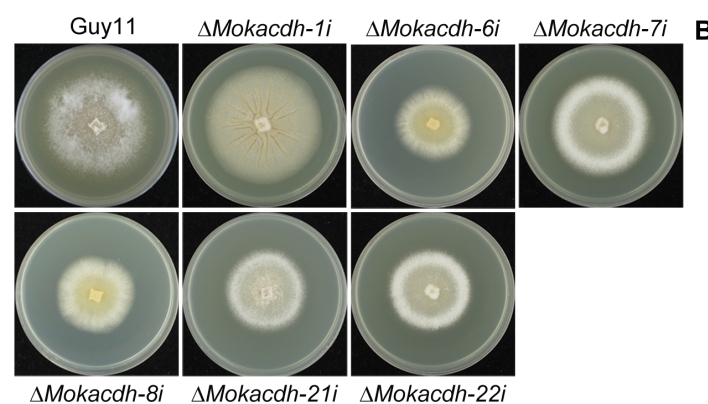

C

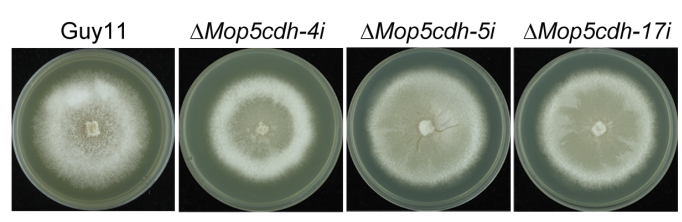

E

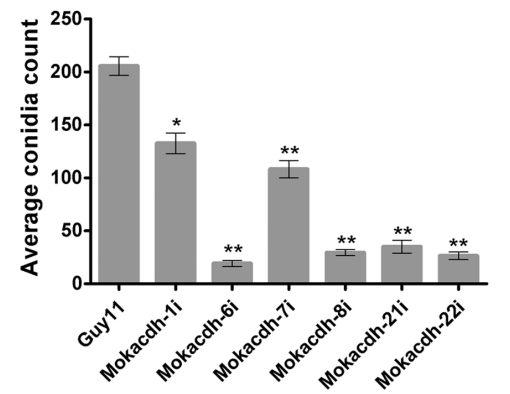

G

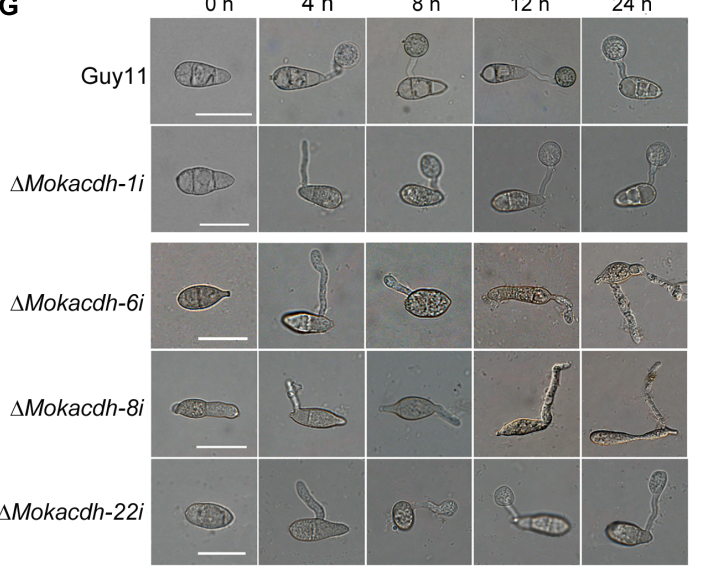

I

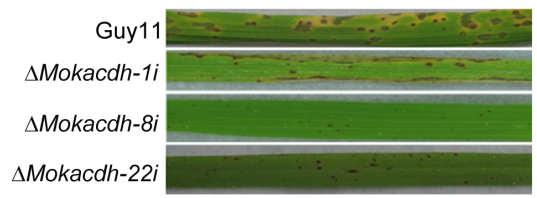

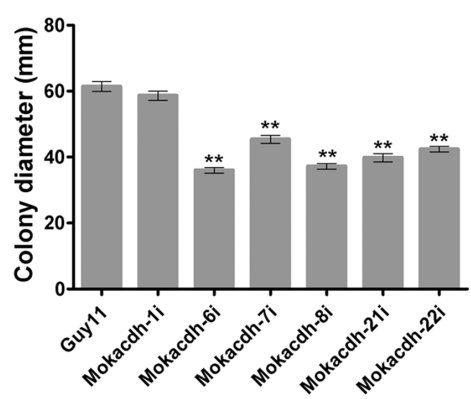

D

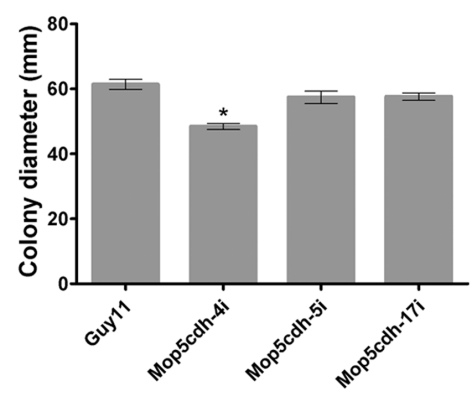

$\mathbf{F}$

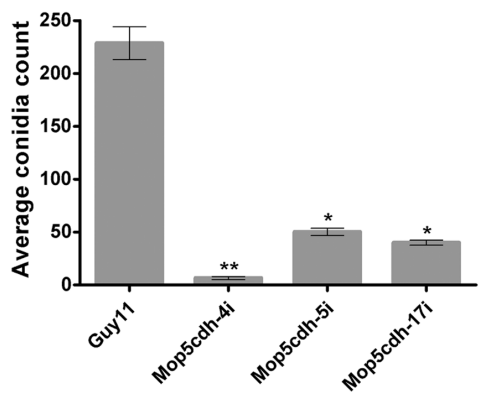

H

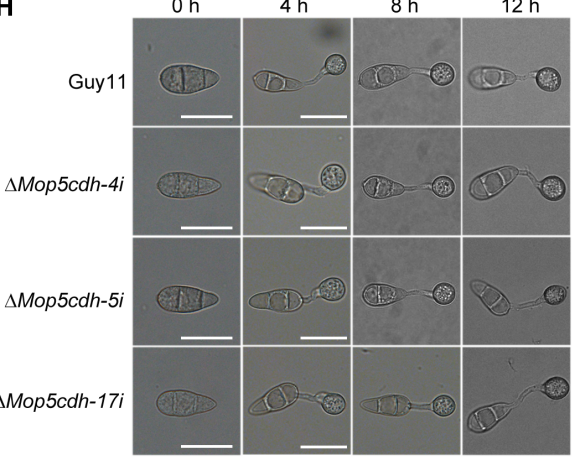

J Guy11

$\Delta M o p 5 c d h-4 i$

$\Delta M o p 5 c d h-5 i$

$\triangle M o p 5 c d h-17 i$

FIGURE 3 | Family-four aldehyde dehydrogenases play indispensable role in survival, growth, sporulation, and pathogenesis of $M$. oryzae. (A) Average colony diameters of $\Delta$ Mokdcdh knock-down strains and the wild-type strain cultured on CM media for 10 days. (B) Statistical evidence of growth defects exhibited by the six independent $\triangle$ Mokdcdh knock-down strains compared with the wild-type strain. (C) The average colony diameters of MoP5CDH knock-down strains and the 
FIGURE 3 | Continued

wild-type strain cultured on CM media for 10 days. (D) The statistical evaluation of the vegetative growth level recorded in the $\Delta M$ Mop5cdh knock-down strains compared with the wild-type strain. (E) Conidiation abilities of the respective $\Delta$ Mokdcdh knock-down strains and the wild-type strain cultured under the same growth conditions. (F) Statistical presentation of conidiation characteristics of the various $\Delta$ Mop5cdh knock-down strains compared with the wild-type strains cultured under similar growth conditions. (G) The different types of conidia abnormalities as well as germination and appressorium abnormalities exhibited by the six independent $\Delta$ Mokdcdh knock-down strains. (H) Morphology conidia, conidia germination, and appressorium formation characteristics exhibited by the three independent MoP5CDH knock-down mutants generated in this study. (I) Results obtained from infection assays conducted by spray inoculation susceptible CO39 rice seedlings with conidia obtained from the six independent $\Delta$ Mokdcdh knock-down strains along with the wild-type conidia as control. ( $\mathbf{J}$ ) Infection capabilities of the three MoP5CDH knock-down strains. Both statistical and nonstatistical experimental data were generated from three independent biological experiments with three replicates each time with consistent results. One-way ANOVA (nonparametric) statistical analysis was carried out with graphpad prism6 and Microsoft Excel spreadsheet, and the error bars represent the standard deviation. Single and double asterisk(s) "** represent significant differences existing between Guy 11 and the respective knock-down mutants $(P<0.05)$ and $(P<0.01)$.

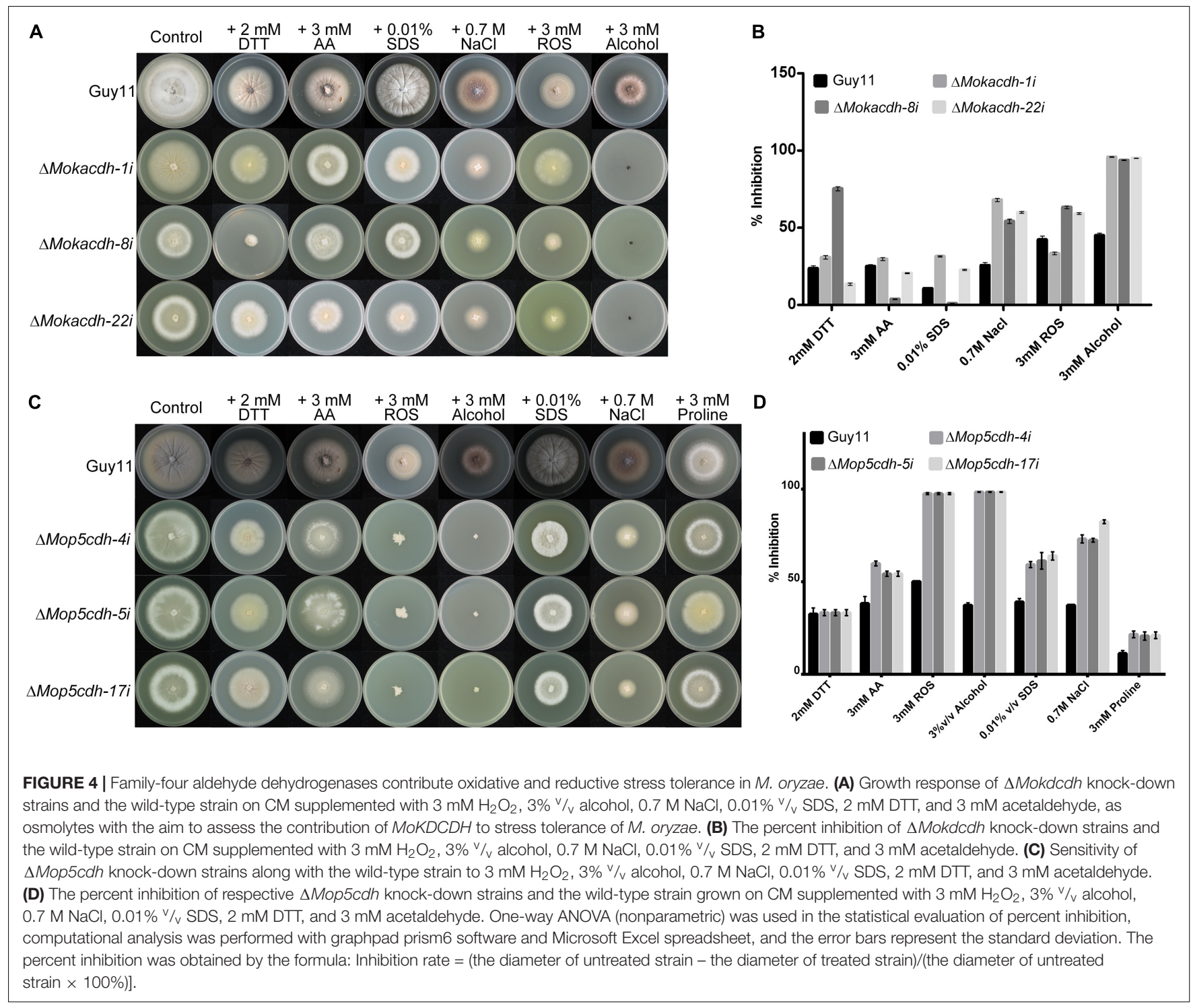

protoplasts were filtered through one-layer sterile Mira-cloth (Calbiochem) and centrifuged at $5000 \mathrm{rpm}$ for $10 \mathrm{~min}$ at $4{ }^{\circ} \mathrm{C}$. The protoplast in pellets was washed twice in $1 \mathrm{M}$ sorbitol STC, re-suspending in STC for final protoplast count under a microscope. Protoplast concentrations were adjusted to $1 \times 106$ and shared into 2 -ml sterilized
Eppendorf tubes at a volume of $250-300 \mu l$ per tube. $7 \%$ DMSO was added to unused protoplast and refrigerated at $-80^{\circ} \mathrm{C}$ for future use. Neomycin-resistant transformants were selected on media supplemented with $200 \mu \mathrm{g} / \mathrm{ml}$ G418 (Invitrogen) and incubated at a steady temperature of $28^{\circ} \mathrm{C}$. 


\section{Construction of Domain Architecture, Phylogenetic Analysis, and Family-Level Classification}

Amino acid sequences of all the 16 ALDHs identified in $M$. oryzae were acquired from the versatile fungi and oomycete genomic resource portal ${ }^{8}$ for the domain prediction. The domain prediction performed with the Pfam domain prediction module and confirmed in the SMART $^{9}$ database. The domain base clustering and phylogenetic analysis of all the 16 M. oryzae aldehyde dehydrogenases were carried out using amino acid sequences of defined Aldedh domain regions. The obtained domain sequences were aligned using Mega version 6 muscle sequence alignment tool using a complete gap deletion approach. The maximum-likelihood method was used to generate the phylogeny. Branches of the tree were tested with 1000 bootstrap replicates. The family-level classification of $M o A L D H s$ was performed through ortholog BLAST and reverse blasting of $M$. oryzae $A L D H s$ (aa) sequences in the human genome database ${ }^{3}$ and $A L D H^{4}$ website, Arabidopsis genome database $^{5}$, and rice genome database ${ }^{6}$ and mapping proteins with more than $90 \%$ identity to their corresponding ortholog families.

\section{Generating ALDHs RNAi Knock-Down Mutants}

$M o K D C D H$ knock-down plasmid vector construct was generated by cloning 108 base pairs (bps) of unique nonhomolog sequence amplified from the $M$. oryzae strain Guy11 cDNA into a pSD1 plasmid digested with restriction enzymes EcoR1 and Xba1. For $M o P 5 C D H$ knock-down mutants, the silencing vector was constructed by cloning 110 bp of unique nonhomologs sequence amplified from the $M$. oryzae strain Guy11 cDNA into the pSD1 plasmid digested with restriction enzymes EcoR1 and Xba1.

\section{Genomic DNA Isolation From Magnaporthe oryzae Using SDS-CTAB Method}

The Guy11 isolates were cultured or grown in liquid media for 4 days in a shaking incubator at a temperature of $27.5^{\circ} \mathrm{C}$ at $120 \mathrm{rpm}$. Mycelia were harvested from the liquid CM by filtering, freeze dried, frozen in liquid nitrogen, and ground into fine powder with a mortar and pestle in liquid nitrogen.

\footnotetext{
${ }^{8}$ http://fungidb.org/fungidb/

${ }^{9}$ http://smart.embl-heidelberg.de/
}

TABLE 2 | Primer pairs used in RT-PCR.

\begin{tabular}{ll}
\hline Gene & Primer sequence \\
\hline MoKDCDH & F: 5' CGTTCGTTGATTGCTTGA 3' \\
R: 5' ATGTGTGGTTGCGTTTAC 3' \\
FoP5CDH & R: 5' GGTCTAAATGAGGGCAAAC 3' \\
MoTUBULIN3 & F: 5' TCTGACTTCAGGAATGGTC 3' \\
& R: 5' AGCGGTCTGGATGTTGTTGG 3'
\end{tabular}

The grounded mycelia were re-suspended in $1 \mathrm{ml}(100 \mu \mathrm{l})$ of ice-cold lysis buffer (150 mM NaCl, $50 \mathrm{mM}$ EDTA, $10 \mathrm{mM}$ Tris$\mathrm{HCl}, \mathrm{pH} 7.4,30 \mu \mathrm{g} / \mathrm{ml}$ proteinase $\mathrm{K}$ ), transferred into a $1.5-\mathrm{ml}$ Eppendorf tube, and kept at $4^{\circ} \mathrm{C}$ to prevent endonuclease activity during rehydration of the sample. SDS was added to a final concentration of $2 \%$, vortexed, and incubated for $30 \mathrm{~min}$ at $65^{\circ} \mathrm{C}$ in a water bath. After centrifugation for $15 \mathrm{~min}$ at $14,000 \mathrm{rpm}$, the supernatant was transferred to a new sterile 1.5-ml Eppendorf tube. The volume of supernatant was measured, and the $\mathrm{NaCl}$ with a concentration of $1.4 \mathrm{M}$ and one-tenth volume of $10 \%$ CTAB buffer (10\% CTAB, 500 mM Tris-HCl, 100 mM EDTA, pH $8)$ were added. The solution was thoroughly mixed and incubated for $10 \mathrm{~min}$ at $65^{\circ} \mathrm{C}$ in a water bath. After cooling for $2 \mathrm{~min}$ at $15^{\circ} \mathrm{C}$, an equal volume of chloroform-isoamyl alcohol $(24: 1 \mathrm{v} / \mathrm{v})$ was added, thoroughly mixed, and the tube was centrifuged for $15 \mathrm{~min}$ at 14,000 rpm. Extraction was repeated until the interface was clear. The supernatant was then pipetted into a new $1.5-\mathrm{ml}$ Eppendorf tube, containing two volumes of cold 100\% ethanol. After DNA precipitation, the pellet was centrifuged for $15 \mathrm{~min}$ at $4^{\circ} \mathrm{C}$ and $14,000 \mathrm{rpm}$. Pellets obtained after centrifugation were washed with $70 \%$ ethanol and dried at room temperature. The resultant product was re-suspended in $100 \mu \mathrm{l}$ TE buffer with $0.002 \%$ RNAse $(5 \mu \mathrm{g} / \mathrm{ml})$ and incubated for $1 \mathrm{~h}$ at $37^{\circ} \mathrm{C}$. The suspension was then used as the template for amplifying required $A L D H$ fragments for intended purposes. The remaining suspension was stored at $-20^{\circ} \mathrm{C}$ for later PCR amplification and other use.

\section{Extracting Total RNA From Magnaporthe oryzae}

The wild-type Guy11 strain and $M o K D C D H$ and $M o P 5 C D H$ knock-down mutants were cultured or grown in the liquid $\mathrm{CM}$ for 4 days in a shaking incubator at a temperature of $27.5^{\circ} \mathrm{C}$ at $12,000 \mathrm{rpm}$. The cultured colonies were then filtered out, washed with sterilized double deionized water, dried, and blended in liquid nitrogen. An equal weight of the grounded samples was transferred into $1.5-\mathrm{ml}$ sterilized Eppendorf tubes, suspended with $1 \mathrm{ml}$ RNAiso, and vortexed vigorously to yield a uniform mixture. The mixture was then placed on the ice briefly and revortexed and allowed to settle for $5 \mathrm{~min}$ at room temperature. $200 \mu \mathrm{l}$ of chloroform was added to the mix and vortexed for $15 \mathrm{~s}$ to get rid of proteins. The contents were allowed to stand still for $3 \mathrm{~min}$ at room temperature before proceeding to centrifugation for $15 \mathrm{~min}$ at $12,000 \mathrm{rpm}$ under $4^{\circ} \mathrm{C} .400 \mu \mathrm{l}$ of the supernatant was pipetted into new sterilized Eppendorf tubes, and $400 \mu \mathrm{l}$ isopropanol was added, gently mixed, and allowed to stand at room temperature for $10 \mathrm{~min}$. The suspension was subjected to centrifugation at $12,000 \mathrm{rpm}$ for $10 \mathrm{~min}$ at $4^{\circ} \mathrm{C}$; the supernatant was discarded leaving the pellets at the bottom. Washing was done by adding $1 \mathrm{ml}$ of $75 \%$ alcohol to the pellets, and centrifugation was done at 12,000 rpm for 5-min at $4^{\circ} \mathrm{C}$, supernatant was discarded after centrifugation, and the precipitates were air dried under room temperature for $5 \mathrm{~min}$. Dried pellets were diluted with RNAse-free water, 10X reaction buffer, and DNAse to prepare an initial solution of $200 \mu \mathrm{l}$ in the ratio of $89: 10: 1$ and incubated for $30 \mathrm{~min}$ at $37^{\circ} \mathrm{C}$. After 
incubation, the solution was heated to a temperature of $65^{\circ} \mathrm{C}$ for $2 \mathrm{~min}$ in a water bath before adding more RNAse-free water to attain a final volume of $500 \mu \mathrm{l}$. An equal volume $(500 \mu \mathrm{l})$ of solution mixture containing water-phenol, chloroform, and isopentanol in the ratio of 25:24:1 was added to the RNA suspension and mixed gently before proceeding to centrifugation at $10,000 \mathrm{rpm}$ for $10 \mathrm{~min}$ at $4^{\circ} \mathrm{C}$. About $200 \mu \mathrm{l}$ of supernatants was pipetted into new sterilized Eppendorf tubes before adding $500 \mu \mathrm{l}$ of absolute alcohol and stored in $-80^{\circ} \mathrm{C}$ for $2 \mathrm{~h}$. The content was then centrifuged for $10 \mathrm{~min}$ at $12,000 \mathrm{rpm}$ at $4^{\circ} \mathrm{C}$ and proceeded to wash by adding $1 \mathrm{ml}$ of $75 \%$ alcohol to the pellets and centrifuged at $12,000 \mathrm{rpm}$ for $5 \mathrm{~min}$ at $4^{\circ} \mathrm{C}$ after which the supernatant was discarded and the precipitates were air dried under room temperature for $5 \mathrm{~min}$. The air-dried precipitates are then eluted with DNA and RNA nucleotide-free water and stored in $-80^{\circ} \mathrm{C}$ till subsequent usage for reverse transcription and for qPCR assays.

\section{Real-Time-PCR}

The expression level of $M o K D C D H$ and $M o P 5 C D H$ in the respective knock-down strains was monitored using quantitative real-time PCR (qRT-PCR). The reverse transcription of RNAs extracted from the respective mutants from $M o K D C D H$, $M o P 5 C D H$ knock-down lines, and the wild-type strain was carried out with SYBR ${ }^{\circledR}$ Premix Ex. Taq ${ }^{\text {TM }}$ (Tli RNaseH Plus) purchase (Takara Biomedical Technology, Beijing Co., Ltd.). A $25 \mu \mathrm{l}$ reaction mix was formulated as follows: 12.5 $\mu$ l Premix Ex-Taq, $1 \mu \mathrm{l}$ of each $10 \mu \mathrm{M}$ forward and reverse primers (Table 2), and $1 \mu$ cDNA template. qRTPCR data were generated by Eppendorf Realplex2 MasterCycler (Eppendorf AG 223341, Hamburg). Data analysis was conducted using delta delta-CT $\left(2^{-\Delta \Delta C t}\right)$ method as described by Livak and Schmittgen (2001) and Zhong et al. (2015) using the expression level of actin as a positive control. The error bars represent mean $\pm \mathrm{SD}$, while single and double asterisks “*” represent a significant reduction in fold expression of $M o K D C D H$ and $M o P 5 C D H$ in their respective knock-down strains. Consistent values were obtained with five independent biological replications and three technical replicates for each independent experiment.

\section{Pathogenicity Assay}

For plant infection assays, conidia were collected from a 7day-old rice-bran medium. Conidial suspensions were adjusted

\section{REFERENCES}

Brocker, C., Vasiliou, M., Carpenter, S., Carpenter, C., Zhang, Y., Wang, X., et al. (2013). Aldehyde dehydrogenase (ALDH) superfamily in plants: gene nomenclature and comparative genomics. Planta 237, 189-210. doi: 10.1007/ s00425-012-1749-0

Bryan, N., Ahswin, H., Smart, N., Bayon, Y., Wohlert, S., and Hunt, J. A. (2012). Reactive oxygen species (ROS)-a family of fate deciding molecules pivotal in constructive inflammation and wound healing. Eur. Cell Mater. 24, 249-265. doi: 10.22203/eCM.v024a18

Buchman, C. D., and Hurley, T. D. (2017). Inhibition of the aldehyde dehydrogenase $1 / 2$ family by psoralen and coumarin derivatives. to $1.5-2.0 \times 105$ conidia $/ \mathrm{ml}$ in $0.02 \%$ Tween solution and sprayed onto 3 - to 4 -week-old susceptible rice seedlings (Oryza sativa cv. CO39). Inoculated plants were incubated in a dark, humid chamber at $25^{\circ} \mathrm{C}$ for $24 \mathrm{~h}$ before being transferred into another humid chamber with 12 -h photoperiod. The plants were examined for disease symptoms after 7 days of post-inoculation (dpi). Consistent results were obtained with five independent biological experiments with three replications.

\section{Appressorium Formation}

Appressorium formation bioassay was conducted by dropping aliquots of $20 \mu \mathrm{l}$ conidia suspension (105) on fisher scientific hydrophobic microscope cover glass and incubated under a humid condition at a temperature of $26^{\circ} \mathrm{C}$ without light. Appressorium development was monitored at every 4-h interval $(4,8,12$, and $24 \mathrm{~h})$ with the aid of an optical microscope or a confocal microscope; at least, 150 conidia per strain/experiment was examined; and abnormalities observed were counted. Both statistical and nonstatistical experimental data were generated from three independent biological experiments with three replicates each time with consistent results.

\section{AUTHOR CONTRIBUTIONS}

ZW, JN, WA, SA, and MS conceived the work, designed the experiments, and wrote the manuscript. WA, SA, LL, XC, FO, TY, and YL conducted the phenotype analysis and checked the off-target effect and the microscope examination.

\section{FUNDING}

This work was supported by the National Key Research and Development Program of China (Grant No. 2016YFD0300707).

\section{SUPPLEMENTARY MATERIAL}

The Supplementary Material for this article can be found online at: https://www.frontiersin.org/articles/10.3389/fpls.2018.00980/ full\#supplementary-material

J. Med. Chem. 60, 2439-2455. doi: 10.1021/acs.jmedchem.6b0 1825

Conrad, K. A., Rodriguez, R., Salcedo, E. C., and Rauceo, J. M. (2018). The Candida albicans stress response gene stomatin-like protein 3 is implicated in ROSinduced apoptotic-like death of yeast phase cells. PLoS One 13:e0192250. doi: 10.1371/journal.pone.0192250

Dong, Y., Liu, H., Zhang, Y., Hu, J., Feng, J., et al. (2017). Comparative genomic study of ALDH gene superfamily in Gossypium: a focus on Gossypium hirsutum under salt stress. PLoS One 12:e0176733. doi: 10.1371/journal.pone.0176733

Feder, M. E., and Hofmann, G. E. (1999). Heat-shock proteins, molecular chaperones, and the stress response: evolutionary and ecological physiology. Annu. Rev. Physiol. 61, 243-282. doi: 10.1146/annurev.physiol.61.1.243 
Furukawa, S., Fujita, T., Shimabukuro, M., Iwaki, M., Yamada, Y., Nakajima, Y., et al. (2017). Increased oxidative stress in obesity and its impact on metabolic syndrome. J. Clin. Invest. 114, 1752-1761. doi: 10.1172/JCI21625

Gill, M. (2014). Pollen storage and viability. Int. J. Bot. Res. 4, 1-18.

Guidarelli, M., Carbone, F., Mourgues, F., Perrotta, G., Rosati, C., Bertolini, P., et al. (2011). Colletotrichum acutatum interactions with unripe and ripe strawberry fruits and differential responses at histological and transcriptional levels. Plant Pathol. 60, 685-697. doi: 10.1111/j.1365-3059.2010.02423.x

Guo, X., Wang, Y., Lu, H., Cai, X., Wang, X., Zhou, Z., et al. (2017). Genomewide characterization and expression analysis of the aldehyde dehydrogenase (ALDH) gene superfamily under abiotic stresses in cotton. Gene 628, 230-245. doi: 10.1016/j.gene.2017.07.034

He, F., and DiMario, P. J. (2011). Drosophila delta-1-pyrroline-5-carboxylate dehydrogenase $(\mathrm{P} 5 \mathrm{CDh})$ is required for proline breakdown and mitochondrial integrity-establishing a fly model for human type II hyperprolinemia. Mitochondrion 11, 397-404. doi: 10.1016/j.mito.2010.12.001

Heller, J., and Tudzynski, P. (2011). Reactive oxygen species in phytopathogenic fungi: signaling, development, and disease. Annu. Rev. Phytopathol. 49, 369-390. doi: 10.1146/annurev-phyto-072910-095355

Hirayama-Kurogi, M., Takizawa, Y., Kunii, Y., Matsumoto, J., Wada, A., Hino, M., et al. (2017). Downregulation of GNA13-ERK network in prefrontal cortex of schizophrenia brain identified by combined focused and targeted quantitative proteomics. J. Proteomics 158, 31-42. doi: 10.1016/j.jprot.2017.02.009

Hossain, M. A., Bhattacharjee, S., Armin, S. M., Qian, P., Xin, W., Li, H. Y., et al. (2015). Hydrogen peroxide priming modulates abiotic oxidative stress tolerance: insights from ROS detoxification and scavenging. Front. Plant Sci. 6:420. doi: 10.3389/fpls.2015.00420

Huang, K., Czymmek, K. J., Caplan, J. L., Sweigard, J. A., and Donofrio, N. M. (2011). HYR1-mediated detoxification of reactive oxygen species is required for full virulence in the rice blast fungus. PLoS Pathog. 7:e1001335. doi: 10.1371/ journal.ppat.1001335

Januchowski, R., Wojtowicz, K., and Zabel, M. (2013). The role of aldehyde dehydrogenase $(\mathrm{ALDH})$ in cancer drug resistance. Biomed. Pharmacother. 67, 669-680. doi: 10.1016/j.biopha.2013.04.005

Jia, B., Jia, X., Kim, K. H., Pu, Z. J., Kang, M. S., and Jeon, C. O. (2017). Evolutionary, computational, and biochemical studies of the salicylaldehyde dehydrogenases in the naphthalene degradation pathway. Sci. Rep. 7:43489. doi: 10.1038/srep43489

Jimenez-Lopez, J. C., Lopez-Valverde, F. J., Robles-Bolivar, P., Lima-Cabello, E., Gachomo, E. W., and Kotchoni, S. O. (2016). Genome-wide identification and functional classification of tomato (Solanum lycopersicum) aldehyde dehydrogenase (ALDH) gene superfamily. PLoS One 11:e0164798. doi: 10.1371/ journal.pone. 0164798

Kirch, H. H., Bartels, D., Wei, Y., Schnable, P. S., and Wood, A. J. (2004). The ALDH gene superfamily of Arabidopsis. Trends Plant Sci. 9, 371-377. doi: 10.1016/j.tplants.2004.06.004

Koop, D. R. (2006). Alcohol metabolism's damaging effects on the cell. Alcohol Res. Health 29, 274-280.

Koštál, V., Šimek, P., Zahradníèková, H., Cimlová, J., and Štìtina, T. (2012). Conversion of the chill susceptible fruit fly larva (Drosophila melanogaster) to a freeze tolerant organism. Proc. Natl. Acad. Sci. U.S.A. 109, 3270-3274. doi: 10.1073/pnas.1119986109

Kotchoni, S. O., Jimenez-Lopez, J. C., Gao, D., Edwards, V., Gachomo, E. W., Margam, V. M., et al. (2010). Modeling-dependent protein characterization of the rice aldehyde dehydrogenase (ALDH) superfamily reveals distinct functional and structural features. PLoS One 5:e11516. doi: 10.1371/journal. pone.0011516

Kotchoni, S. O., Kuhns, C., Ditzer, A., Kirch, H. H., and Bartels, D. (2006). Overexpression of different aldehyde dehydrogenase genes in Arabidopsis thaliana confers tolerance to abiotic stress and protects plants against lipid peroxidation and oxidative stress. Plant Cell Environ. 29, 1033-1048. doi: 10.1111/j.13653040.2005.01458.x

Lehmann, S., Serrano, M., L'haridon, F., Tjamos, S. E., and Metraux, J. P. (2015). Reactive oxygen species and plant resistance to fungal pathogens. Phytochemistry 112, 54-62. doi: 10.1016/j.phytochem.2014.08.027

Li, Z., Wang, J., Long, H., Zeng, Y., Mei, F., Chen, H., et al. (2017). Cloning and expression analysis of an aldehyde dehydrogenase gene from
Camellia oleifera. Nanosci. Nanotechnol. Lett. 9, 364-373. doi: 10.1166/nnl.2017. 2340

Livak, K. J., and Schmittgen, T. D. (2001). Analysis of relative gene expression

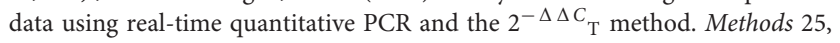
402-408. doi: 10.1006/meth.2001.1262

Luo, M., Gates, K. S., Henzl, M. T., and Tanner, J. J. (2015). Diethylaminobenzaldehyde is a covalent, irreversible inactivator of ALDH7A1. ACS Chem. Biol. 10, 693-697. doi: 10.1021/cb500977q

Missihoun, T. D., Kotchoni, S. O., and Bartels, D. (2018). Aldehyde dehydrogenases function in the homeostasis of pyridine nucleotides in Arabidopsis thaliana. Sci. Rep. 8:2936. doi: 10.1038/s41598-018-21202-6

Morgan, C. A., and Hurley, T. D. (2015). Characterization of two distinct structural classes of selective aldehyde dehydrogenase $1 \mathrm{~A} 1$ inhibitors. J. Med. Chem. 58, 1964-1975. doi: 10.1021/jm501900s

Muñoz-Clares, R. A., González-Segura, L., Murillo-Melo, D. S., and RiverosRosas, H. (2017). Mechanisms of protection against irreversible oxidation of the catalytic cysteine of ALDH enzymes: possible role of vicinal cysteines. Chem. Biol. Interact. 276, 52-64. doi: 10.1016/j.cbi.2017. 02.007

Norvienyeku, J., Zhong, Z., Lin, L., Dang, X., Chen, M., Lin, X., et al. (2017). Methylmalonate-semialdehyde dehydrogenase mediated metabolite homeostasis essentially regulate conidiation, polarized germination and pathogenesis in Magnaporthe oryzae. Environ. Microbiol. 19, 4256-4277. doi: $10.1111 / 1462-2920.13888$

Palukaitis, P., and Carr, J. (2008). Plant resistance responses to viruses. J. Plant Pathol. 90, 153-171.

Pérez-Arellano, I., Carmona-Álvarez, F., Martínez, A. I., Rodríguez-Díaz, J., and Cervera, J. (2010). Pyrroline-5-carboxylate synthase and proline biosynthesis: from osmotolerance to rare metabolic disease. Protein Sci. 19, 372-382. doi: $10.1002 /$ pro. 340

Perozich, J., Nicholas, H., Wang, B. C., Lindahl, R., and Hempel, J. (1999). Relationships within the aldehyde dehydrogenase extended family. Protein Sci. 8, 137-146. doi: 10.1110/ps.8.1.137

Ranf, S., Eschen-Lippold, L., Pecher, P., Lee, J., and Scheel, D. (2011). Interplay between calcium signalling and early signalling elements during defence responses to microbe-or damage-associated molecular patterns. Plant J. 68, 100-113. doi: 10.1111/j.1365-313X.2011.04671.x

Schnitzer, E., Pinchuk, I., and Lichtenberg, D. (2007). Peroxidation of liposomal lipids. Eur. Biophys. J. 36, 499-515. doi: 10.1007/s00249-007-0146-2

Singh, S., Brocker, C., Koppaka, V., Chen, Y., Jackson, B. C., Matsumoto, A., et al. (2013). Aldehyde dehydrogenases in cellular responses to oxidative/electrophilicstress. Free Radic. Biol. Med. 56, 89-101. doi: 10.1016/j.freeradbiomed.2012.11.010

Smith, J. M., and Heese, A. (2014). Rapid bioassay to measure early reactive oxygen species production in Arabidopsis leave tissue in response to living Pseudomonas syringae. Plant Methods 10:6. doi: 10.1186/1746-4811-10-6

Stiti, N., Missihoun, T. D., Kotchoni, S., Kirch, H. H., and Bartels, D. (2011). Aldehyde dehydrogenases in Arabidopsis thaliana: biochemical requirements, metabolic pathways, and functional analysis. Front. Plant Sci. 2:65. doi: 10.3389/ fpls.2011.00065

Thannickal, V. J., and Fanburg, B. L. (2000). Reactive oxygen species in cell signaling. Am. J. Physiol. Lung Cell. Mol. Physiol. 279, L1005-L1028. doi: 10. 1152/ajplung.2000.279.6.L1005

Trotter, E. W., and Grant, C. M. (2002). Thioredoxins are required for protection against a reductive stress in the yeast Saccharomyces cerevisiae. Mol. Microbiol. 46, 869-878. doi: 10.1046/j.1365-2958.2002.03216.x

Vaidyanathan, H., Sivakumar, P., Chakrabarty, R., and Thomas, G. (2003). Scavenging of reactive oxygen species in NaCl-stressed rice (Oryza sativa L.)differential response in salt-tolerant and sensitive varieties. Plant Sci. 165, 1411-1418. doi: 10.1016/j.plantsci.2003.08.005

Valadi, H., Valadi, Å., Ansell, R., Gustafsson, L., Adler, L., Norbeck, J., et al. (2004). NADH-reductive stress in Saccharomyces cerevisiae induces the expression of the minor isoform of glyceraldehyde-3-phosphate dehydrogenase (TDH1). Curr. Genet. 45, 90-95. doi: 10.1007/s00294-003-0469-1

Vasiliou, V., Pappa, A., and Estey, T. (2004). Role of human aldehyde dehydrogenases in endobiotic and xenobiotic metabolism. Drug Metab. Rev. 36, 279-299. doi: 10.1081/DMR-120034001 
Wan, P. J., Fu, K. Y., Lü, F. G., Wang, X. X., Guo, W. C., and Li, G. Q. (2015). Knocking down a putative $\Delta 1$-pyrroline-5-carboxylate dehydrogenase gene by RNA interference inhibits flight and causes adult lethality in the Colorado potato beetle Leptinotarsa decemlineata (Say). Pest Manag. Sci. 71, 1387-1396. doi: $10.1002 / \mathrm{ps} .3941$

Wood, A. J., and Duff, R. J. (2009). The aldehyde dehydrogenase (ALDH) gene superfamily of the moss Physcomitrella patens and the algae Chlamydomonas reinhardtii and Ostreococcus tauri. Bryologist 112, 1-11. doi: 10.1639/00072745-112.1.1

Yao, Z., Zou, C., Zhou, H., Wang, J., Lu, L., Li, Y., et al. (2013). $\Delta 1$ Pyrroline-5-carboxylate/glutamate biogenesis is required for fungal virulence and sporulation. PLoS One 8:e73483. doi: 10.1371/journal.pone.0073483

Zhang, Y., Mao, L., Wang, H., Brocker, C., Yin, X., Vasiliou, V., et al. (2012). Genome-wide identification and analysis of grape aldehyde dehydrogenase (ALDH) gene superfamily. PLoS One 7:e32153. doi: 10.1371/journal.pone. 0032153

Zhong, Z., Norvienyeku, J., Yu, J., Chen, M., Cai, R., Hong, Y., et al. (2015). Two different subcellular-localized Acetoacetyl-CoA acetyltransferases differentiate diverse functions in Magnaporthe oryzae. Fungal Genet. Biol. 83, 58-67. doi: 10.1016/j.fgb.2015.08.008

Zhu, C., Ming, C., Zhao-Shi, X., Lian-Cheng, L., Xue-Ping, C., and You-Zhi, M. (2014). Characteristics and expression patterns of the aldehyde dehydrogenase (ALDH) gene superfamily of foxtail millet (Setaria italica L.). PloS One 9:e101136. doi: 10.1371/journal.pone.0101136

Conflict of Interest Statement: The authors declare that the research was conducted in the absence of any commercial or financial relationships that could be construed as a potential conflict of interest.

Copyright (C) 2018 Abdul, Aliyu, Lin, Sekete, Chen, Otieno, Yang, Lin, Norvienyeku and Wang. This is an open-access article distributed under the terms of the Creative Commons Attribution License (CC BY). The use, distribution or reproduction in other forums is permitted, provided the original author(s) and the copyright owner(s) are credited and that the original publication in this journal is cited, in accordance with accepted academic practice. No use, distribution or reproduction is permitted which does not comply with these terms. 